\title{
PROPAGATION OF ELASTIC WAVES
}

\author{
G. J. COOPER AND J. W. CRAGGS \\ (Received 28 October 1964, revised 23 August 1965)
}

\begin{abstract}
The behaviour of waves in elastic solids with linear stress strain curves is expressed, for plane strain, by a pair of simultaneous partial differential equations of hyperbolic type. Detailed behaviour of the waves is examined by solving these equations numerically.

\section{Introduction}

One of the important unsettled questions in connection with the strength of materials is to what extent the yield stress, and hence the plastic behaviour, of a metal depends on rate of strain. The answer to this question may be sought by the analysis of tests at high rates of loading. These tests are most easily carried out by ballistic means, either by detonating a slab of explosive against the face of a plate [Pack, Evans and James (1948)] or by shooting a projectile at a thin wire [Talylor (1940)] or by shooting through the bore of a thin cylinder, at a plug at the far end.

Experiments of the first type have a considerable advantage, in that they produce, effectively, a uni-axial strain, and so are easily analysed mathematically. The problems of instrumentation are such, however, that only a fragmentary picture of the stress strain situation in the material can be obtained. Much fuller information can be obtained in the second and particularly, in the third types of experiment, for strain gauges can be used to give a time trace of surface strain at each of a number of points. Bell (1956), (1961a), (1961b)] shoots a rod at an identical specimen on which are ruled diffraction gratings. Davies (1948) uses an electrical capacitance technique to carry out measurements on a rod. The data from these types of experiment are more difficult to analyse since the problem of waves on a rod or wire is basically a three dimensional problem. Investigators have rarely attempted exact analysis, being content to use a one dimensional model, assuming uni-axial stress. Such assumption is valid for harmonic disturbances of long wave length but not necessarily for the ballistic problems invisaged here and for elastic waves it has been
\end{abstract}


shown [see e.g. Love (1931)] that the uni-axial theory of wave propagation in a rod becomes progressively more inaccurate as the rate of stress increases. Davies (1948) obtained agreement between experimental results and a theoretical analysis which allowed for dispersion. Shalak (1957) has obtained theoretical results for large time which compare well with the work of Davies.

As a first step towards a complete analysis of waves in plastic solids we attempted to determine the behaviour of waves in elastic solids. We restricted the analysis to two dimensions and to materials with linear stress strain curves. With these assumptions, the behaviour may be determined by a pair of simultaneous partial differential equations of hyperbolic type. These differential equations are replaced by difference equations which are solved numerically by a process involving stepping. ahead in two directions. A rectangular mesh is chosen for simplicity and the interval size taken sufficiently small to guard against growth of rounding errors. The error growth has been examined by a Mesh-Fourier process, and some tests have been carried out on the final results, indicating stability of the numerical process.

It is believed that a two dimensional analysis should be sufficient to indicate the type of behaviour occurring in rods. It shoulds be possible to extend the analysis to include non-linear stress strain curves and also to include plastic behaviour.

\section{Derivation of the differential equations}

The problem to be considered is the behaviour of an isotropic elastic body subjected to a constant velocity shock. The body will be assumed to be a plate bounded in a Cartesian co-ordinate system by $y= \pm a, x=0$, and unbounded in $z$ and the positive $x$ direction. The shock is in the direction of increasing $x$.

The stress strain relations are [Sokolnikoff (1946), p. 70]

$$
E e_{i j}=(1+v) \sigma_{i j}-v \delta_{i j} \sigma_{i i}, \quad \begin{aligned}
E & \equiv \text { Young's modulus, } \\
\nu & \equiv \text { Poisson's ratio, }
\end{aligned}
$$

where the $e_{i j}$ are the strain components and the $\sigma_{i j}$ are the stress components.

The dynamical equations may be written [Sokolnikoff (1946), p. 82]

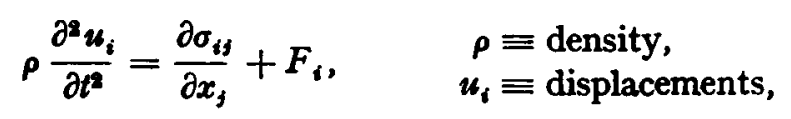

and the body forces $F_{a}$ are zero for an initially unstressed solid. For the plate being considered these tensor equations reduce, in Cartesian co-ordinates, to: 


$$
\begin{aligned}
& E e_{x x}=\sigma_{x x}-v \sigma_{y y}-v \sigma_{z z}, \quad e_{x x}=\frac{\partial u}{\partial x} \\
& E e_{y y}=-\nu \sigma_{x y}+\sigma_{y y}-\nu \sigma_{z x}, \quad e_{y y}=\frac{\partial v}{\partial y} ; \\
& E e_{z z}=-v \sigma_{x x}-v \sigma_{y y}+\sigma_{z z}, \quad e_{z z}=0 ; \\
& \frac{E}{1+\nu} e_{x \nu}=\sigma_{x y} \text {, } \\
& e_{x y}=\frac{1}{2}\left(\frac{\partial u}{\partial y}+\frac{\partial v}{\partial x}\right) \\
& \frac{E}{1+\nu} e_{y z}=\sigma_{y z} \text {, } \\
& e_{v z}=\frac{1}{2}\left(\frac{\partial v}{\partial z}+\frac{\partial w}{\partial y}\right) \\
& \frac{E}{1+\nu} e_{2 x}=\sigma_{x x} \text {, } \\
& e_{z x}=\frac{1}{2}\left(\frac{\partial w}{\partial x}+\frac{\partial u}{\partial z}\right)
\end{aligned}
$$

where $u, v, w$ represent the displacement in the $x, y, z$ directions and:

$$
\begin{aligned}
& \rho \frac{\partial^{2} u}{\partial t^{2}}=\frac{\partial \sigma_{x x}}{\partial x}+\frac{\partial \sigma_{y x}}{\partial y}+\frac{\partial \sigma_{z x}}{\partial z} \\
& \rho \frac{\partial^{2} v}{\partial t^{2}}=\frac{\partial \sigma_{z y}}{\partial x}+\frac{\partial \sigma_{y y}}{\partial y}+\frac{\partial \sigma_{z y}}{\partial z}
\end{aligned}
$$

For plane strain $e_{s s}=0, \sigma_{x z}=\sigma_{s y}=0$, and so from (1), (2)

$$
\begin{aligned}
& E \frac{\partial u}{\partial x}=(1+v)\left[(1-v) \sigma_{x x x}-v \sigma_{y y}\right], \\
& E \frac{\partial v}{\partial y}=(1+v)\left[(1-v) \sigma_{y y}-v \sigma_{x x}\right] .
\end{aligned}
$$

Combining we obtain

$$
\begin{aligned}
& \sigma_{m u}=\frac{E}{(1+v)(1-2 v)}\left[(1-v) \frac{\partial u}{\partial x}+v \frac{\partial v}{\partial y}\right], \\
& \sigma_{v y}=\frac{E}{(1+v)(1-2 v)}\left[(1-v) \frac{\partial v}{\partial y}+v \frac{\partial u}{\partial x}\right],
\end{aligned}
$$

and we also have

$$
\sigma_{x \nu}=\frac{E}{2(1+v)}\left[\frac{\partial u}{\partial y}+\frac{\partial v}{\partial x}\right] .
$$

Scaling the co-ordinate system by a factor $E /(1+v)$ and substituting (5), $(6),(7)$ in the dynamic equations (3) and (4) we have 


$$
\begin{aligned}
& (1-2 v) \frac{\partial^{2} u}{\partial t^{2}}=(1-v) \frac{\partial^{2} u}{\partial x^{2}}+\frac{1}{2} \frac{\partial^{2} v}{\partial x \partial y}+\frac{1-2 v}{2} \frac{\partial^{2} u}{\partial y^{2}} \\
& (1-2 v) \frac{\partial^{2} v}{\partial t^{2}}=\frac{1-2 v}{2} \frac{\partial^{2} v}{\partial x^{2}}+\frac{1}{2} \frac{\partial^{2} u}{\partial x \partial y}+(1-v) \frac{\partial^{2} v}{\partial y^{2}}
\end{aligned}
$$

with the new time scale $t_{\text {new }}=\sqrt{E / \rho(1+v)} t_{\text {original }}$.

These equations can be written in the form

$$
(1-2 v) \frac{\partial^{2}}{\partial t^{2}}\left[\frac{\partial u}{\partial x}+\frac{\partial v}{\partial y}\right]=(1-v) \nabla^{2}\left(\frac{\partial u}{\partial x}+\frac{\partial v}{\partial y}\right)
$$

and another equation in $\partial u / \partial y-\partial v / \partial x$. These are wave equations with wave velocities $c=\sqrt{(1-v) /(1-2 v)}$ and a smaller wave velocity for the second equation.

For a plate bounded by $x=0, y= \pm a$ we have for boundary conditions, for $y= \pm a, t \geqq 0, x \geqq 0$ :

$$
\begin{aligned}
& \sigma_{x y}=0, \text { that is, } \frac{\partial u}{\partial y}=-\frac{\partial v}{\partial x}, \\
& \sigma_{y y}=0, \text { that is, } \frac{\partial u}{\partial x}=-\frac{1-\nu}{\nu} \frac{\partial v}{\partial y},
\end{aligned}
$$

and for $x=0, t \geqq 0$

$$
\frac{\partial u}{\partial t}=U, \quad \frac{\partial v}{\partial t}=0
$$

$U$ a constant.

The initial conditions are $u, v, \partial u / \partial t, \partial v / \partial t$ everywhere zero for $t=0$, $x>0$. The end conditions are such that the plate moves with constant velocity in the $x$ direction.

\section{Difference equations for main points}

Consider steps in time $\delta t, t=j \delta t, j=0,1,2, \cdots$, and at each time interval consider the $x, y$ plane divided into a rectangular mesh of intervals

$$
\begin{aligned}
& \delta x, x=k \delta x, \\
& k=0,1,2, \cdots \text {, } \\
& \delta y, y=l \delta y \text {, } \\
& l=0,1,2, \cdots 7 \text {. }
\end{aligned}
$$

The number of steps in $y$ has been restricted for simplicity of programming. The finite difference approximations used are generally available in any text on numerical analysis [e.g. Milne (1949), p. 96, p. 191]. The approximation used for the mixed derivative is given in Buckingham (1957), p. 505 , or can be easily derived. Substituting the simplest available central difference formulae for the derivatives in (8), (9) we obtain 


$$
\begin{aligned}
u_{j+1, k, l}= & 2 u_{j, k, l}\left[1-\frac{1-v}{1-2 v}\left(\frac{\delta t}{\delta x}\right)^{2}-\frac{1}{2}\left(\frac{\delta t}{\delta y}\right)^{2}\right]-u_{j-1, k, l} \\
& +\frac{1-v}{1-2 v}\left(\frac{\delta t}{\delta x}\right)^{2}\left[u_{j, k+1, l}+u_{j, k-1, l}\right] \\
& +\frac{1}{2}\left(\frac{\delta t}{\delta y}\right)^{2}\left[u_{j, k, l+1}+u_{j, k, l-1}\right] \\
& +\frac{1}{8(1-2 v)} \frac{(\delta t)^{2}}{\delta x \delta y}\left[v_{j, k+1, l+1}-v_{j, k+1, l-1}-v_{j, k-1, l+1}+v_{j, k-1, l-1}\right] \\
& +O\left[(\delta t)^{2}\left\{(\delta x)^{2}+(\delta y)^{2}+(t \delta)^{2}\right\}\right]
\end{aligned}
$$

and we can also obtain a second equation with $\boldsymbol{u}$ and $\boldsymbol{x}$ interchanged with $v$ and $y$ respectively. These two equations enable the calculation of the $u, v$ displacements for the time interval $j+1$ at all points of the $x, y$ mesh if we are given the displacements at all points of the mesh for the $j, j-l$ time intervals.

Let us choose $l=[(1-v) /(1-2 v)](\delta t / \delta x)^{2}+\frac{1}{2}(\delta t / \delta y)^{2}$. We can choose $\delta x=\delta y$ since this should still give sufficient information. Then for $v<\frac{1}{2}$ we have $c \delta t<\delta x$ where $c$ is the fast wave velocity. This indicates a likely exponential decay of rounding errors accumulated at any stage.

The final equations for displacements at the main points of the plate become

$$
\begin{aligned}
u_{j+1, k, l}= & -u_{g-1, k, l}+u_{j, k+1, l}+u_{j, k-1, l} \\
& +(1-2 v)\left[u_{j, k, l+1}+u_{j, k, l-1}-u_{j, k+1, l}-u_{j, k-1, l}\right] \\
& +\frac{1}{4}\left[v_{j, k+1, l+1}-v_{j, k+1, l-1}-v_{j, k-1, l+1}+v_{j, k-1, l-1}\right] \\
& +O(\delta x)^{4}
\end{aligned}
$$

and a similar equation with $u, v$ and $k, l$ interchanged.

\section{Boundary and initial conditions}

The behaviour of the waves is symmetric about the $y=0$ axis and (13) reduces to:

and also

$$
\begin{aligned}
u_{j+1, k, 0}=- & u_{j-1, k, 0}+u_{j, k+1,0}+u_{j, k-1,0} \\
& +(1-2 v)\left[2 u_{j, k, l}-u_{j, k+1,0}-u_{j, k-1,0}\right] \\
& +\frac{1}{2}\left[v_{j, k+1, l}-v_{j, k-1, l}\right]+O(\delta x)^{k}
\end{aligned}
$$

$$
v_{j+1, k, 0}=0
$$


As a result of this symmetry the equations need only be solved for one half of the plate.

On the boundary $y=+a$ conditions (10), (11) hold. A variety of ways of including these equations were considered with the combined problem of accuracy, speed of computation and simplicity of programming. It was finally decided to incorporate these equations in the main equations which hold right up to the boundary. The equations (10), (11) hold only on the boundary and hence expressions for $\partial^{2} u / \partial y^{2}$ in the main equations cannot be substituted for by differentiating the boundary conditions with respect to $y$. We can, however, transform, say, $\partial^{2} v / \partial x \partial y$ to $-v /(1-v) \partial^{2} u / d x^{2}$ and then substitute a central difference approximation for $\partial^{2} u / \partial x^{2}$. $\partial^{2} u / \partial y^{2}$ is evaluated by a Taylor series expansion

and

$$
\left.\left.\frac{(\delta y)^{2}}{2 !} \frac{\partial^{2} u}{\partial y^{2}}\right]_{j, k, a}=u_{j, k, a-1}-u_{j, k, a}+\delta y \cdot \frac{\partial u}{\partial y}\right]_{j, k, a}+O(\delta y)^{2}
$$

$$
\left.\left.\frac{\partial u}{\partial y}\right]_{1, k, a}=-\frac{\partial v}{\partial x}\right]_{1, k, a}
$$

and similarly for $\partial^{2} v / \partial y^{2}$. Thus we obtain for the boundary points, again taking $1=[(1-v) /(1-2 v)](\delta t / \delta x)^{2}+\frac{1}{2}(\delta t / \delta y)^{2}$,

$$
\begin{aligned}
u_{j+1, k, a}= & -u_{j-1, k, a}+\frac{v}{(1-2 v)(1-v)}\left(\frac{\delta t}{\delta x}\right)^{2} u_{j, k, a}+\left(\frac{\delta t}{\delta x}\right)^{2} u_{j, k, a-1} \\
& +\left[\frac{1-v}{1-2 v}-\frac{v}{2(1-2 v)(1-v)}\right]\left(\frac{\delta t}{\delta x}\right)^{2}\left[u_{j, k+1, a}+u_{j, k-1, a}\right] \\
& +\frac{1}{2}\left(\frac{\delta t}{\delta x}\right)^{2}\left[-v_{j, k+1, a}+v_{j, k-1, a}\right]+O\left(\delta x^{3}\right) \\
v_{j+1, k, a}= & -v_{j-1, k, a}+v_{j, k, a-1} \\
& \left.+\left[1-\left(\frac{\delta t}{\delta x}\right)^{2}\right] 2 v_{j, k, a}+v_{s, k, a-1}\right] \\
& +\frac{v}{1-2 v}\left(\frac{\delta t}{\delta x}\right)^{2}\left[-v_{j, k+1, a}-v_{j, k-1, a}\right] \\
& +\frac{v}{1-2 v}\left(\frac{\delta t}{\delta x}\right)^{2}\left[-u_{j, k+1, a}+u_{j, k-1, a}\right]+O\left(\delta x^{3}\right) .
\end{aligned}
$$

The initial conditions may be described quite simply. At $j=0$ the $u, v$ displacements are everywhere zero and $\partial u / \partial t=U, \partial v / \partial t=0$ at $k=0$ for all $j$. At $j=1$ the effect of the shock will not have reached the $k=1$ interval and the above formulae will give correct results only if the 
$j=0, j=-1$ planes are zero at the start of the program. At $k=0$; for all $j$, we can evaluate the displacements as

$$
u=U j \delta t, \quad v=0 .
$$

\section{Program}

The computations were carried out on the Ferranti Pegasus Mk. 2 digital computer installed at Leeds University. This machine has a small high speed store and a large backing store. Program and data in the backing store must be transferred to the high speed store before use and transfers to and from the backing store can be carried out in blocks of eight 39 bit words. It was decided to restrict the number of $y$ intervals to seven so that associated with each $x$ interval are two blocks, one for $u$ displacements, one for $v$ displacements. Due to symmetry, this gives a total of 14 intervals across the plate.

All displacements for the last two time planes, $j, j-1$, evaluated are stored in the backing store. When the displacements for time interval $j+1$ are calculated they are stored in place of the $j-1$ time plane.

We took $U=1$ and chose $\delta x$ so small that for the number of time intervals to be considered $u_{j, 0,1} \ll 1$ and hence all displacements are considerably less than one and there is no danger of overflow when working with fixed point arithmetic. $\delta x$ was chosen as 0.0001 (it was thought that after 100 time intervals the wave behaviour would be settled) so that $\delta t=0.0001 /$ $\sqrt{ } 2$. Poisson's ratio $v$ was chosen as 0.25 .

The program is divided into three parts. The first section clears the whole of the store, reads in the parameters $y, \delta x$, number of $y$ intervals, and some print parameters and stores them and calculates constants required for the difference equations. The second part deals with the evaluation of all the difference equations and has been fitted entirely into the high speed store. For one time interval it evaluates the $u, v$ displacements for all the $y$ intervals (including boundary points) for successive $x$ intervals until all the displacements for an $x$ interval are zero. The third section, the print subroutine, is then called in to decide what information, if any, is to be printed and to output it in a suitable format. Control is then returned to the second section which proceeds to evaluate the displacements for the next time interval. The time required to compute the first 100 time intervals, for six $y$ intervals, was approximately one hour. Of this time about 30 minutes was taken up with the output of results on a slow paper tape punch.

\section{Results}

Initially displacements were printed at every third $x$ interval along the 


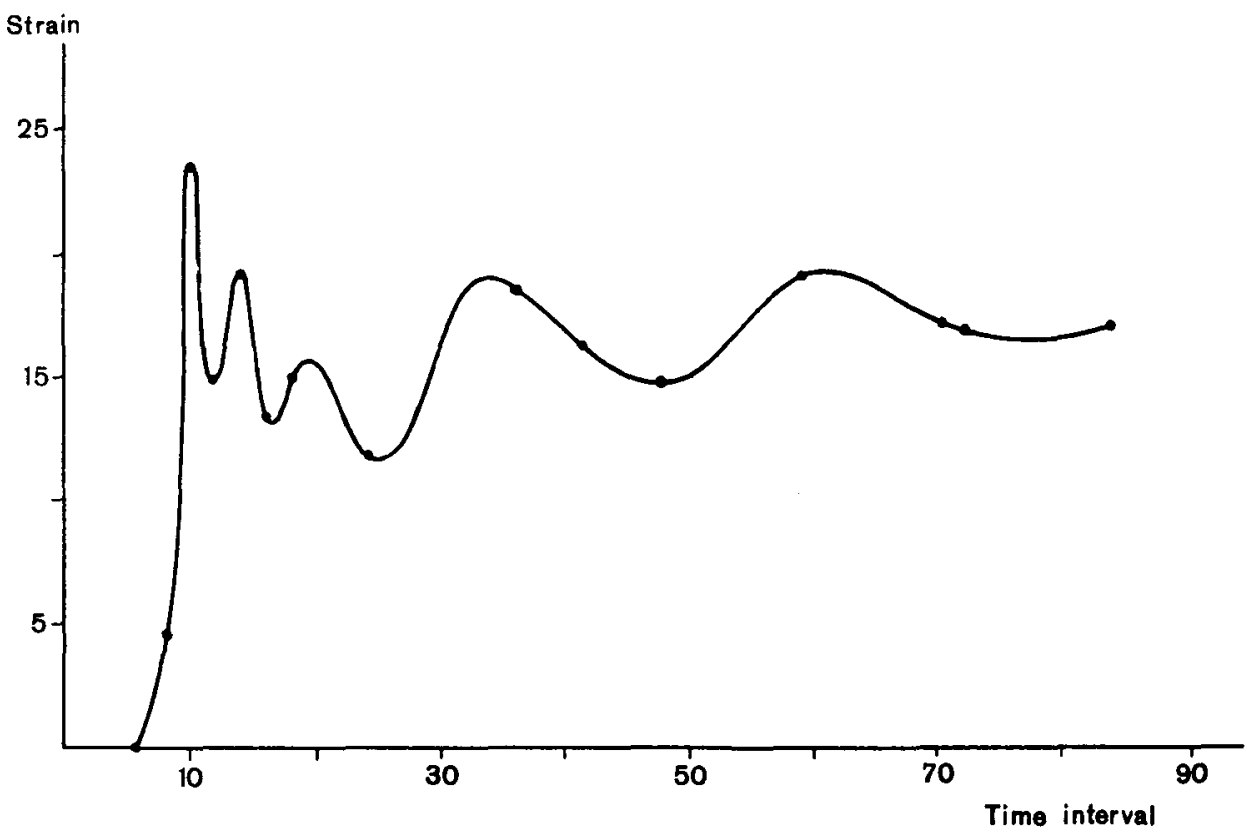

Figure 1

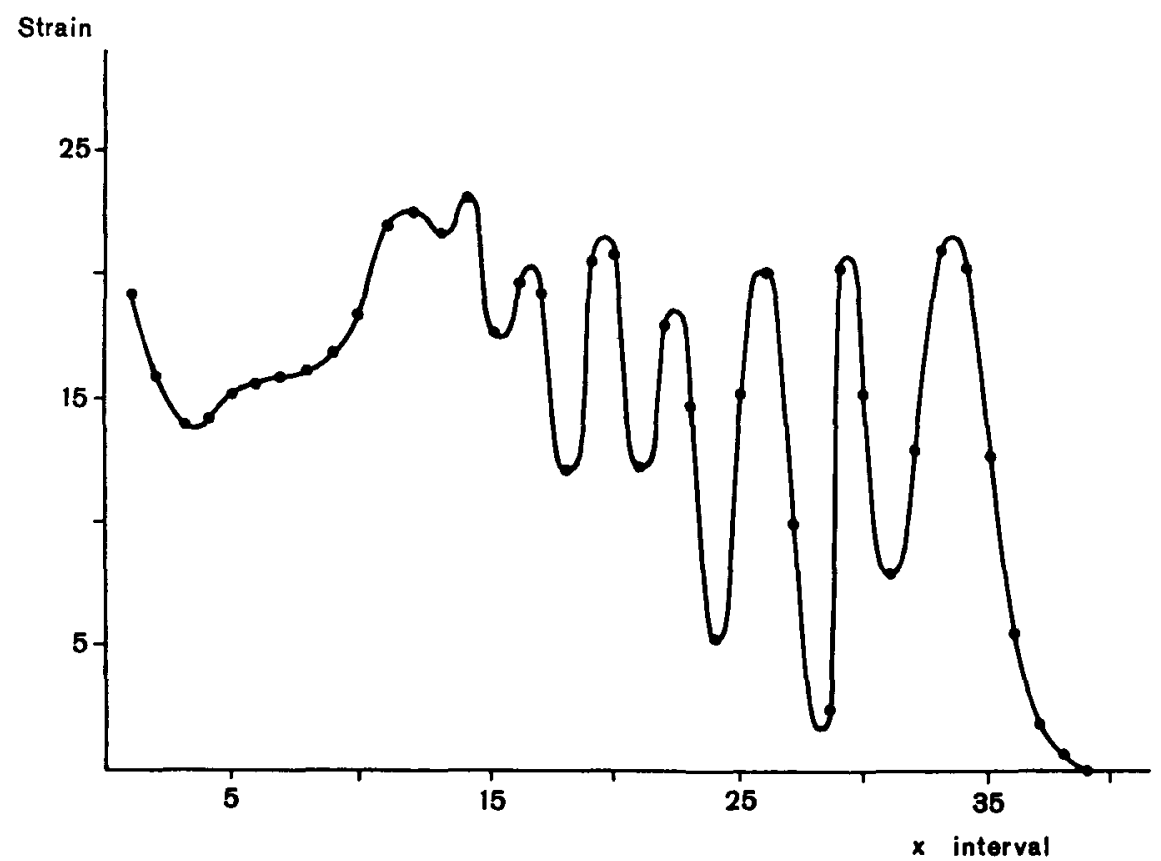

Figure 2 
boundary, for every fourth interval in time. Corresponding displacements at points in the plate were also printed. At a later stage considerably more detail was printed for the boundary to allow an accurate determination of the strain, $\delta u / \delta x$. In Figure 1 the strain has been plotted against increasing time at about half a plate width from the impact face. The waves appear to settle down satisfactorily and this alone indicates that rounding errors are not propagated through the system. One time interval represents approximately $0.059 a / c$ seconds where $a$ is the plate width in inches and $c$ is the velocity of sound in inches/second in the material being considered. For aluminium and a plate of width one inch this is about $0.4 \mu \mathrm{s}$ and for a material such as steel, with elastic behaviour at much higher impact velocities, this does not vary markedly. In Figure 2 the strain has been plotted against increasing distance at 42 time intervals after impact.

The results illustrated in Figure 1 agree qualitatively with data obtained by Davies (1948) and with the theoretical results of Shalak (1957). The results indicated in Figure 2 appear rather irregular but appear to have been confirmed by recent additional results, obtained using a program based on an alternative formulation of the problem. With this program it has also

TABLE OF DIFFERENCES IN $\boldsymbol{x}$

\begin{tabular}{rrr}
\hline$k$ & Displacement $u$ & First difference \\
\hline 0 & 0.00254558 & \\
1 & 0.00245048 & 0.00009510 \\
2 & 0.00237159 & 0.00007889 \\
3 & 0.00228971 & 0.00008188 \\
4 & 0.00220408 & 0.00008563 \\
5 & 0.00212423 & 0.00007985 \\
6 & 0.00203605 & 0.00008818 \\
7 & 0.00194993 & 0.00008612 \\
8 & 0.00185065 & 0.00009928 \\
9 & 0.00175111 & 0.00009954 \\
10 & 0.00162800 & 0.00012311 \\
11 & 0.00153067 & 0.00009743 \\
12 & 0.00143256 & 0.00009801 \\
13 & 0.00131830 & 0.00011426 \\
14 & 0.00126772 & 0.00005058 \\
15 & 0.00115680 & 0.00011092 \\
16 & 0.00108153 & 0.00007527 \\
17 & 0.00105313 & 0.00002840 \\
18 & 0.00095706 & 0.00009607 \\
19 & 0.00089958 & 0.00005748 \\
20 & 0.00087146 & 0.00002812 \\
21 & 0.00076162 & 0.00011984 \\
22 & 0.00066745 & 0.00008417 \\
23 & 0.00064668 & 0.00002077 \\
24 & 0.00056546 & 0.00009122 \\
\hline & & \\
\hline & &
\end{tabular}


been possible to vary the ratio $\delta x / \delta y$, results obtained being in excellent agreement with those presented here. Checks have been obtained by differencing the numerical data with respect to the time and $x$ coordinates. Differences seem to be satisfactorily small. The table gives first differences with respect to $x$ for $j=36$. The differences are constant to four decimal places and indicate that sufficient accuracy has been obtained up to one diameter from the impact face. Further away the numerical solution has still to settle down.

\section{References}

Bell, J. F., 1956, Journ. App. Physics, 27, 10

Bell, J. F., 1961a, b, J. Mech. Phys. Solids, 9, pp 1-15, 261-278.

Buckingham, R. A. 1957, Numerical Methods, Pitman, London.

Davies, R. M., 1948, Phil. Trans. Roy. Soc. London, A, $240,375$.

Love, A. E. H., 1931, Mathematical Theory of Elasticity, 4th Ed., C.U.P. Cambridge, p. 428. Milne, E. W., 1949, Numerical Calculus, Princeton University Press, Princeton.

Pack, D. C., W. M. Evans and D. H. James, 1948, Proc. Phys. Soc., 61, 1.

Shalak, R., 1957, J. App. Mech., 24, 59.

Sokolnikoff, I. S., 1946, Mathematical Theory of Elasticity, McGraw-Hill, New York.

Taylor, G. I. 1940, unpublished. 